\title{
Simultaneous Multiobjective Multiple Route Selection Using Genetic Algorithm for Car Navigation
}

\author{
Basabi Chakraborty \\ Faculty of Software and Information Science, \\ Iwate Prefectural University, \\ 152-52 Aza Sugo, Takizawamura, Iwate 020-0193, Japan \\ basabi@soft.iwate-pu.ac.jp
}

\begin{abstract}
A genetic algorithm (GA) based multiple route selection for car navigation device is proposed in this work. The proposed scheme offers the driver a choice from alternate near optimal solutions, each of which carries some specific characteristics based on the knowledge of the road map and the environment. The algorithm has been simulated on some real road map and it is found that it provides better solution compared to deterministic algorithms and other GA based algorithms in terms of driver's satisfaction.
\end{abstract}

\section{Introduction}

One of the essential component of a car navigation system is route planning. Given a set of origin-destination pair, there could be many possible routes for a driver. Search for optimal route from one point to another on a weighted graph is a well known problem and has several solutions. Though these algorithms can produce stable solutions in polynomial time, they exhibit high computational complexity specially in changing real time environment. In case of car navigation the shortest path may not be the best one from other considerations such as, simplicity, traffic congestion, environmental problem or simply user's satisfaction. So for an efficient practical car navigation device in dynamic environment, we need to specify multiple and different good (near optimal) choices accroding to multiple different criteria which make the search space too large to find out the solution in real time by deterministic algorithms.

A few research works on automated route selection for navigation are reported in literature [1] 2]. Genetic algorithms (GA) are now widely used to solve search problems with applications in practical routing and optimization problems [3]. GA includes a variety of quasi optimal solutions which can be obtained in a given time. Some works 4] [5] have also been reported for search of multiple routes for car navigation systems using GA. The problem in finding multiple semi optimal routes simultaneously is that the selected routes resemble each other i,e they partly overlap. Inagaki et.al [5] proposed an algorithm to minimize the effect of overlapping solutions. But the proposed algorithm requires a large 
solution space to attain high quality solution due to its inconsistent crossover mechanism. Inoue [6] proposed a method for finding out multiple different (non overlapping) routes by dividing the road map in multiple areas and putting different weights in each of them so that the selected routes are through different areas of the map. But as their is no direct method for comparing the overlapping of the selected paths this method is not guaranteed to select minimally overlapped multiple semi optimal paths. The author has previously proposed in [7] a GA based algorithm for finding out multiple nonoverlapping routes simultaneously while the selected routes did not possess any specific characteristics (i,e the objective of selection is not known) to be chosen by the driver. In this work a scheme for multiobjective multiple route selection on the basis of some specific characteristics of the route for car navigation using GA has been proposed. In the proposed method a set of nonoverlapping optimal routes are selected on the basis of different known criterion so that the driver can decide easily according to his choice. The proposed algorithm has been evaluated by simulation experiment with a piece of real road map and compared with Dijkustra and other GA based algorithms for multiple route selection.

\section{Multiobjective Multiple Route Selection by GA}

Genetic algorithm consists of three steps, problem coding, design of evaluation function and genetic operators (reproduction, crossover and mutation). The proposed GA is described below.

\subsection{Problem Coding}

The road map is first converted into a connected graph, considering each road crossing as a node $(n)$ in the graph. The roads in map are represented by links $(\mathrm{L})$ in the graph. The information of the road map is stored in a knowledge base in the form of the node numbers of the connecting node pair and a n-dimensional connecting link vector $\left(L=\left[l_{1}, l_{2} \ldots l_{n}\right]\right)$ whose components represent requisite knowledge regarding different choice of path such as the distance between the connecting node pair, the information of turnings (left or right), the information whether the link passes through mountain or by the side of the river etc. for every pair of connecting nodes. Any possible path from start node to goal node via other nodes is a possible solution and coded as a chromosome by using node numbers. However looping in the path is avoided.Population of chromosomes representing solution paths (routes) are generated by genetic coding. Chromosomes are equal length sequence of integers where each gene represents a node number.

\subsection{Design of Fitness Function}

In this work the fitness function has been designed with the objective of providing a set of alternate solution of routes with some knowledge about the individual routes such as the route passing through the side of a river, the route passing through mountain or the route has minimum number of turning or minimum 
number of signal. The knowledge based multiple choices of route will provide the driver better satisfaction and reasoning capability for selection of route.

The general fitness function $F$ is defined here as follows:

$$
F=\Sigma_{1}^{n} f_{i}\left(l_{i}\right)
$$

where $f_{i}\left(l_{i}\right)$ represents a function of the $i$ th component of link vector. The idea is that the selection of optimum path according to $i$ th choice of criterion will basically depend on the fitness function based on $l_{i}$, the requisite information regarding $i$ th choice. Thus the design of $f_{i}$ will depend on $l_{i}$. So depending on the choice of criteria, the value of the fitness function of same chromosome will be different. Here lower value of $F$ corresponds to better solution.

\subsection{Proposed Algorithm}

The algorithm for finding out a set of solution containing ' $\mathrm{m}$ ' alternate near optimal routes according to ' $\mathrm{m}$ ' different criteria is as follows:

1. Initial population is formed by random selection of paths (chromosomes) from the entire solution space.

2. The individual chromosomes are evaluated multiply by the different component of the fitness function corresponding to different choices of criterion and ' $\mathrm{m}$ ' group of chromosomes are formed by ranking corresponding to ' $\mathrm{m}$ ' alternate selection. Any particular chromosome may belong to two groups. 'm' different groups are then subjected to genetic operation as follows:

(a) For each group, the first few chromosomes ( number to be selected according to the problem, we used 2 in our simulation) are retained as it is (elite selection) for the next generation.

(b) For other chromosomes in the group crossover operation with probability $P_{c}$ has been done with parents selected by Roulette selection with the group specific fitness function. The crossover position is selected at the place where the node numbers of the parents match.

(c) Mutation with a very small probability $P_{m}$ at a random position is done to generate new population in the group.

(d) All the groups are then put together to form the next generation of population. The new population is then checked for duplicate chromosome and looping of path.

3. The new population is then ranked again by evaluating with the different fitness function and the procedure is repeated.(Go to step 2)

4. The stopping criterion is decided beforehand as a trade off between the availability of computation time and goodness of the produced solution.

5 . The best route of each group ' $\mathrm{m}$ ' after final iteration is selected to form ' $\mathrm{m}$ ' alternate solutions.

\section{Simulation Experiment}

The simulation experiment has been done on a real road map. The road map is first converted into a graph with 130 nodes. The number of candidate paths from 
start $(\mathrm{X})$ to destination node $(\mathrm{Y})$ are found to be 100 ( no looping is considered). The optimal path for different choices are considered in this experiment as 1) shortest route depending on total distance 2) optimal route with minimum number of turnings 3) optimal route through mountain and 4) optimal route passing by the side of a river. The link vector $\mathrm{L}$ with the road information has been calculated and stored as the knowledge base. The fitness function for different criteria $\left(f_{i}\right)^{\prime}$ 's of Eq.(1) are designed as follows:

$$
f_{1}\left(l_{1}\right)=\Sigma_{1}^{n} \text { rlength }(i)
$$

where rlength $(i)$ represents the distance of the path between $n$th and $n-1$ node, the number of nodes in the path being $n$.

$$
f_{2}\left(l_{2}\right)=\Sigma_{1}^{n} \text { rlength }(i)+\Sigma P R+\Sigma P L
$$

$\Sigma P R$ and $\Sigma P L$ denotes the penalty for right and left turn respectively.

For choice 3 and 4 , the following criterion function has been designed where $C<1$ and $l(3)=1$ represents that the node passes through the desired path of choice.

$$
\begin{aligned}
f_{3}\left(l_{3}\right) & =\Sigma_{1}^{n} \text { rlength }(i) \times(2-C) \text { if } l(3)=0 \\
& =\Sigma_{1}^{n} \text { rlength }(i) \times C \text { if } l(3)=1
\end{aligned}
$$

$C$ is problem dependent and set heuristically. We chose $C=0.2$ for this problem. The algorithm has been run for several times.

Dijkstra's, Inagaki's method and author's earlier proposed method have also been used for simulation with same road map for comparison. The selected genetic parameters are represented in Table 1 . In both the methods the parameters $P_{c}$ and $P_{m}$ have been changed to several values and the optimum values are noted in the table.

Table 1. Setting of parameters of GA Table 2. Comparative Performance of different Algorithms

\begin{tabular}{|c|c|c|c|c|c|}
\hline Method & $\begin{array}{c}\text { Population } \\
\text { size }\end{array}$ & $\begin{array}{c}\text { No. of } \\
\text { iteration }\end{array}$ & $P_{c}$ & $P_{m}$ & $P_{g}$ \\
\hline Inagaki & 100 & 40 & 0.9 & 0.5 & \\
\hline Earlier & 150 & 40 & 0.8 & 0.6 & 0.2 \\
\hline Proposed & 150 & 40 & 0.8 & 0.2 & \\
\hline
\end{tabular}

\begin{tabular}{|c|c|c|}
\hline Algorithm & Time taken & $\begin{array}{c}\text { Average. No. of } \\
\text { overlapping } \\
\text { nodes }\end{array}$ \\
\hline Dijkstra & $.014 \mathrm{~s}$ & 15 \\
\hline Inagaki & $.182 \mathrm{~s}$ & 5 \\
\hline Earlier & $.177 \mathrm{~s}$ & 2 \\
\hline Proposed & $.167 \mathrm{~s}$ & 4 \\
\hline
\end{tabular}

\section{Simulation Results}

Table 2. shows the comparative results of the different algorithms. Dijkstra algorithm takes much shorter time compared to other algorithms for finding out the 


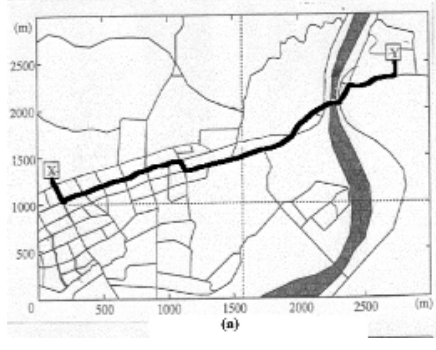

Fig. 1. Route by shortest distance

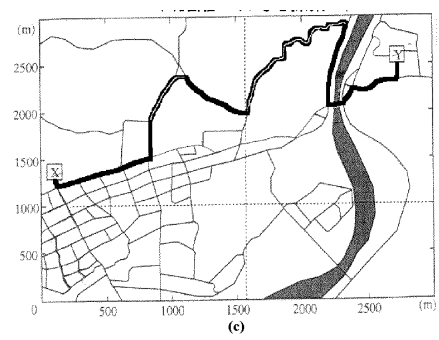

Fig. 3. Route through mountain

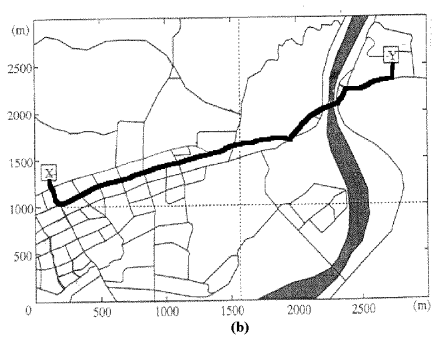

Fig. 2. Route by minimum turninng

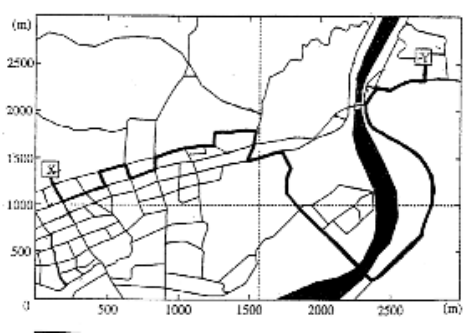

Fig. 4. Route by the side of river

shortest route and it also is able to find out better paths in terms of distance. But successive short routes are highly overlapped. Both Inagaki's method and the algorithms proposed by author's earlier and in this paper take longer time than Dijkstra's algorithm but alternate routes can be found out simultaneously. The proposed algorithms by author is found to be better than Inagaki's method in terms of nonoverlapping. In the newly proposed method only route 1 and route 2 has overlapping in between them but route 3 and route 4 are non overlapping with all others. The proposed algorithm in this work is also better than the earlier proposed algorithm in terms of the complexity and computation time of GA. One of the parameter $P_{g}$ is not needed in the newly proposed algorithm. The alternate routes according to distance, number of turns, passing through mountain and passing by the side of river are found out and are shown. Though this simulation could not produce the best (optimal) routes the four alternate routes are quite independent and not much overlapping. As they satisfy some physical criterion based on the knowledge of the map the driver can use better reasoning capability while choosing from the alternatives.

\section{Conclusion}

In this work a Genetic Algorithm based solution technique for finding out $m$ routes simultaneously with different characteristics for car navigation has been proposed. Simultaneous multiple route selection is difficult by popular optimization technique like Dijkstra algorithm. Currently available GA based algorithm 
can produce multiple routes simultaneously but selected routes resemble each other. Author has proposed a GA based technique in an earlier work for finding out multiple routes simultaneously with minimal overlapping by grouping $m$ routes as one set of solution and designing fitness functions in such a way that it penalizes the function for overlapping. But though the algorithm produces better routes in terms of minimal overlapping, the alternate routes do not carry any meaning. It will be helpful to the driver to take decision and thereby get more satisfaction if we can provide the alternate routes with some special characteristics. In this work a GA based algorithm for finding multiple alternate routes have been proposed in which the fitness function is designed in such a way that the generated routes provide optimality according to some specific criteria and therefore satisfy the driver with some special knowledge about the generated routes. The simulation results with a real road map shows non optimality of some of the routes and the design of the fitness function depends on the map itself. Several experiments with different road maps are to be done to remove these two drawbacks. But from this limited experiment it is found that the alternate routes provide better satisfaction to the driver with a better computation time and complexity than other currently available methods.

\section{References}

1. Shapiro, J. et al., "Level Graphs and Approximate Shortest Paths Algorithms", Networks, Vol 22, pp. 691-717, 1992.

2. Liu, B., "Intelligent Route Finding: Combining Knowledge, Cases and an Efficient Search Algorithm", in Proceedings of 12th European Conference on Artificial Intelligence, pp.380-384, 1996.

3. Ahn, C. W. and Ramakrishna, R. S. , "A Genetic Algorithm for Shortest Path Routing Problem and the Sizing of Populations", IEEE Trans. on Evolutionary Computations, Vol. 6, no. 6,pp. 566-579, December 2002.

4. Kanoh, H. and Nakamura, T.,Knowledge based Genetic Algorithm for Dynamic Route Selection, in Proceedings of International Conference on Knowledge based Intelligent engineering Systems and Allied Technologies, pp. 616-619, 2000.

5. Inagaki, J. et, al.,A method of Determining Various Solutions for Routing Application with a Genetic Algorithm,in Trans of IEICE, J82-D-I, No.8. pp.11 02-1111, August 2002.(in Japanese)

6. Inoue, Y., Exploration Method of Various Routes with Genetic Algorithm, Master's Thesis,Information System Engineering, Kochi Institute of Technology, 2001.(in Japanese)

7. Chakraborty, B.,'GA-based Multiple Route Selection for Car Navigation', Lecture Notes in Computer Science (Proceedings of AACC 2004), LNCS 3285 Springer, pp. 599-605, 2004. 